Proceedings

\title{
Close2U: An App for Monitoring Cancer Patients with a Gamification System to Improve the Engagement ${ }^{\dagger}$
}

\author{
Javier Navarro-Alamán ${ }^{1, *}$, Raquel Lacuesta ${ }^{1,2}$, Iván García-Magariño ${ }^{3}$ and Jesús Gallardo ${ }^{1}$ \\ 1 Department of Computer Science and System Engineering, University of Zaragoza, 50009 Zaragoza, Spain; \\ lacuesta@unizar.es (R.L.); jesus.gallardo@unizar.es (J.G.) \\ 2 Instituto de Investigación Sanitaria Aragón, University of Zaragoza, 50009 Zaragoza, Spain \\ 3 Department of Software Engineering and Artificial Intelligence, Complutense University of Madrid, \\ 28040 Madrid, Spain; igarciam@ucm.es \\ * Correspondence: jnavarroa@unizar.es; Tel.: +34-978-618-102 \\ + Presented at the 13th International Conference on Ubiquitous Computing and Ambient Intelligence \\ UCAmI 2019, Toledo, Spain, 2-5 December 2019.
}

Published: 21 November 2019

\begin{abstract}
Nowadays, gamification offers several advantages in order to motivate a change in the behavior towards health and wellness. Although it is a relatively new trend, many fields have already realized its potential, and those related to health have also begun to make use of it. This paper introduces an application developed to improve patient monitoring and motivation through the use of gamification. We have applied the mechanics and dynamics of games in a non-game context, such as the introduction of data for health monitoring, in order to attract the patient. With the use of gamification, we make the introduction of data less tedious and, in addition, increase levels of motivation, as a further benefit. In this work we have conducted a user study aimed at evaluating the usability of gamification. We also studied the resources that encourage patients to use the application and how to increase their motivation and satisfaction. The results show that the app is easy to use. Second, they show that we implemented a scalable and self-recursive system. Finally, these results indicate that our system for resources sharing is a system in which patients feel comfortable when sharing and receiving those resources and they encourage us for further developments and studies based on the feedback received.
\end{abstract}

Keywords: gamification; social interaction; health; cancer; motivation; resources

\section{Introduction}

Gamification is a term that refers to the use of video game elements in non-gaming systems in order to get a better user experience (UX) and user engagement [1]. It refers to the use of game mechanics in non-play environments and applications in order to enhance motivation, concentration, effort and loyalty, among others, and is about establishing a strategy to influence and motivate groups of people. Gamification is structured on the mechanics and dynamics of games.

There has been considerable interest in applying gamification in the digital healthcare industry. This growing interest was to some extent spawned by the inequity of access to healthcare resources, the lack of adherence to treatment and the increase in healthcare costs [2]. Although gamification is increasingly used in interventions, evidence on its effectiveness remains sparse [3]. However, in a review of the literature for gamification in health [4], Johnson shows results in which $59 \%$ are positive and $41 \%$ mixed or neutral. A positive note is that several studies found evidence that applications with gamification oriented to health directly increased the well-being of the participants. When the end users are cancer patients, an evaluation of all the possible influential factors in the evolution of 
applications using gamification is fundamental. Furthermore, the need to monitor oncological patients is considered a fundamental aspect in their follow-up [5].

In some studies, such as $[4,6]$, it is shown that gamification could have a positive effect on health and wellbeing, improving them in a wide variety of areas, for a variety of sociodemographic groups, especially when applied in a skilled way. The evidence is stronger for the use of gamification to target behavioral outcomes, particularly physical activity, and weakest for its impact on cognition. There is also an initial support for gamification as a tool to support other physical health related outcomes, including nutrition and medication use, as well as mental health outcomes, including wellbeing, personal growth, flourishing, stress and anxiety.

The objective in this paper is to integrate gamification in an application for the follow-up of cancer patients, which allows the registering of treatments and medication and includes surveys to know how they feel each day. We can understand that monitoring the feeling of patients by means of an application is one of a duty or obligation. Therefore, in this work we have added gamification to give more dynamism to the patient and have tried to mitigate the monotony and the repetition of having to do the same action every day. We have applied the mechanics and the dynamics of a game to our application, in order to make these obligations less tedious and less of a burden.

The rest of the paper is structured as follows. Section 2 analyzes the related work. The Close2U application is described in Section 3. Section 4 analyzes the methods that we use in this research. Section 5 presents the results and discussion. Finally, our conclusions and suggestions for future work are given in Section 6.

\section{Related Work}

We have carried out a study of applications in which gamification has helped patients and in turn allowed advances and discoveries in medicine. In [7] it can be found a review of some popular taxonomies to identify the common strategies listed by some authors. After the study, Cugelman identifies six core ingredients of gamification, with the exception of fun and playfulness in the ambit of health, because it is important not to ignore this and keep it in mind, although it is something that has not received much attention in the health behavior change literature. These six mechanics of gamification are: goals, challenges, feedback, reinforcement, progress and social. Furthermore, to know better about the users of gamification, Marczewski proposed six user types that differ in the degree to which they can be motivated by either intrinsic or extrinsic motivational factors [8]: philanthropists, socializers, free spirits, achievers, players and disruptors.

PhytoCloud is a mobile web application that focuses on breast cancer patients and which enables users to record their dietary habits and physical activity and motivates them to consume food with estrogen-like properties. This allows them to control their own goals and motivates them to achieve them and adopt healthier eating habits, while also increasing physical activity, in order to ensure an improvement in their lifestyle and wellbeing [9].

The Pain Squad application collects information about pain suffered and the effects that cancer treatments have on children. This task must be done twice a day so that the collected data can be taken into account in research. In the case of children, it is usually a very tedious task. The game follows a theme in which a police patrol must solve a crime. The children answer a series of questions through a smartphone application, whereby when they finish, they receive a reward or a prize that they must collect. There is also the possibility of promotion within the patrol and the user can go up in rank. Each completed report helps the team to solve the case. In addition, it includes some videos in which real agents congratulate the user for their achievement [10].

To help all types of patients in their day-to-day treatment, we have studied the application Mango Health. This application counts the number of times the patient remembers taking their medication and rewards the patient for it financially. The application sends alerts at the moment at which the patient must fulfill their obligation in the treatment, and after taking their medication, they are rewarded by obtaining points. If they maintain their commitment for a certain time without error, these points translate into up to 5 or 10 dollars in gift cards or, depending on the type of target, 
donations can even be made to charities. In addition, it shows the progress made in the treatment, the benefits it brings, a history and what remains for the patient to do in order to their reward [11].

The objective of Reflexion Health is to, as its motto says, "reinvent the experience of physical therapy". Ravi Komatireddy is also a co-founder of 'Reflexion Health', the company that owns the project, and he was the one who detected a problem when treating his patients in that there is a lack of continuity and commitment on the part of the patients during physical therapies. They developed a virtual platform with instructions for use at home, which is watched through a television or any type of image player, and an animated instructor shows the exercise to be performed. The application asks the patient to follow the exercises by copying the avatar that appears on the screen, and to verify that the therapy is actually being performed, the program has a 'Kinect' motion capture technology [12] which compares the performance of the patient with that as required by the pre-configured virtual therapist. The platform tracks the patient and sends information to the doctor, with the necessary checks performed in real time or deferred, to review their progress and be corrected if necessary [13].

In Table 1 we can see the six mechanics of gamification from the study of Cugelman [7] compared with the aforementioned applications. It can be seen that none of the applications meet all the gamification mechanics.

Table 1. The six mechanics of gamification compared with the applications studied.

\begin{tabular}{ccccc}
\hline & Pain Squad & Mango Health & Reflexion Health & PhytoCloud \\
\hline Goals & Yes & Yes & Yes & Yes \\
Challenges & No & Yes & Yes & Yes \\
Feedback & No & No & Yes & No \\
Reinforcement & Yes & Yes & No & No \\
Progress & Yes & Yes & Yes & Yes \\
Social & Yes ${ }^{1}$ & No & No & Yes ${ }^{1}$ \\
\hline \multicolumn{5}{c}{ ( Only share the classification with all users. }
\end{tabular}

In conclusion, according to the aforementioned works, gamification has been useful to improve some health applications. This motivates the inclusion of gamification in the development of the Close2U application. This app can provide analyses to physicians for helping them to improve the treatment of patients. The novelty of the Close2U application is to include gamification filling the gap of the literature of the social component about connecting cancer patients with each other, to improve the motivation and experience of patients. Thus, this app generates a social environment of participation rewarding the sharing of resources by patients. These resources (e.g. songs) should make other cancer patients feel good. A correct implementation of gamification strategies [14] allows to go from mere connectivity to commitment, achieving that any type of collective participate in a dynamic and proactive way in actions that generally require an effort of the will.

\section{The Close2U Application}

This section presents the app specification that was agreed after several meetings with patients and psychologists, performing usability tests, such as Cognitive Walkthrough. Three psychologists from the AECC (Spanish Association against Cancer) in Teruel and two cancer patients were present throughout the design and development process. We improved the app with their feedback, following a user-centered design. For example, we incorporated motivation exercises and their tracking thanks to the users' feedback.

To make the whole system easy to use, the user interface has been conceived from a UserCentered Design perspective. The application focuses on all types of cancer patients, taking both psychological and emotional aspects into account, reflecting the experience in the collaboration with AECC. 
Section 3.1 presents the app specification, section 3.2 introduces the system architecture, and section 3.3 describes the user interface, focusing the gamification part.

\subsection{App specification}

In the app, patients shall be able to create, update, delete and see the details of their medication and appointments. Also, they shall be able to complete two diary surveys, having the option to complete them or not, since we do not want the patient to feel it as an obligation.

The gamification system allows the patients to share resources with the other patients, not as a "social network" itself, but as a network with which patients interact with each other but in an anonymous way.

The rewards for patients are their own shared motivations, being validated by the psychologists before being introduced them into the reward system. We wanted that the content that a patient can share is not harmful for other patients or is able to demotivate them. The problem would be not only that they would not use the application, but also something more serious, since it could affect them emotionally and cause damage in their way of thinking or his way of dealing with the disease. We must not forget that we are dealing with very specific users, which are cancer patients.

To get rewards, patients should complete the daily survey. They always get a motivating phrase and can choose another reward. They can choose between a motivating resource, an exercise or not getting anything. The patients shall be able to rate the phrases and motivational resources on a scale of 1 to 5 . The patient shall also be able to perform an exercise, previously created by the psychologists with some steps to make and some repetitions.

The patient has a personal points classification, in which he gains experience every time he performs actions in the app. The total points correspond to a classification (a) From 0 to 5000 points, Bronze (b) From 5001 to 10,000 points, Silver and (c) From 10,001, Gold.

Each time they perform one of the following actions the patient receives points: Conduct a survey, create an appointment, modify an appointment, create a medication, modify a medication, upload a reward, get the reward accepted by the psychologist, assess a reward received, and perform an exercise. All the actions before mentioned has a value of 50 points.

We want to reward the patient whenever they share something, even if they share something that is sensitive for other patients. In this way, they are intended to motivate them, since the other resources they share can be good for other patients, and so we do not punish or cut something that we want to encourage. If some problem is detected in a very popular resource, the psychologists will be in charge of telling the patient something related to that resource or why he has done it. On the other hand, if the resource is suitable, we will give the patient more points in order to reward him.

The resources raised by the patients have to keep a control, so that they could not share things that could be harmful to other patients. The psychologists can check the assessment that patients have given the resource, and if a resource is ranked very low by other patients, the psychologist will value if is necessary to deactivate it, if they think it is harmful for other patients. The psychologists will be able to make custom exercises for the patients, which can have the subject that psychologists think is convenient.

\subsection{System Architecture}

Figure 1 shows the Close2U system architecture. As it can be seen, for the front-end for both applications, the mobile application and the web application, we use one of the most succesful and known software architecture patterns, the Model View Controller (MVC) pattern. Hence, when the modules and functionality are expanded or changed, only the modified parts will be affected and the others will be able to function as before without making any substantial changes. We believe that this is essential to correctly perform a user-centered design. The web front-end is built as a templated CodeIgniter website that communicates with the API and the mobile front-end is build in Java and XML. XML is used only for the view of the application, but not for data, which is always managed in JSON. 


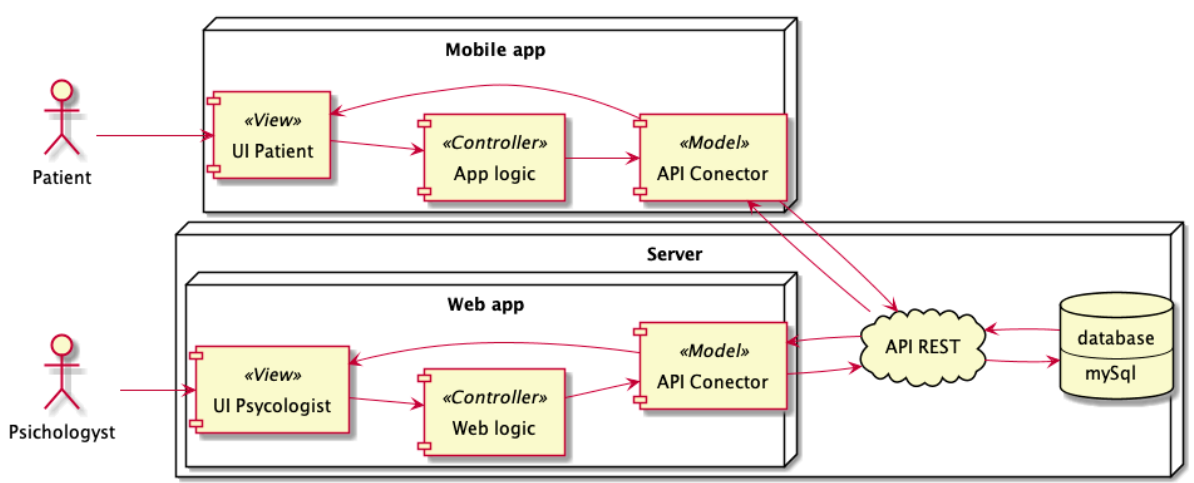

Figure 1. Close2U system architecture

The back-end application programming interface (API) is an MVC structured RESTful JSON API built with PHP, aided by the framework CodeIgniter. The database driver is MySQL and it makes https requests to connect the API with the database.

\subsection{User Interface}

Figure 2a shows the screen that appears after completing a survey. A motivational phrase will appear, and the user can evaluate it from 1 to 5 . The system tells the patient that a reward is available for completing the survey and give his three options, (a) motivational resource, (b) exercise and (c) nothing. On the motivation screen a motivation item that has been shared by another patient is shown to the patient with a name and description as we show in Figure $2 b$. This item will be able to be rated from 1 to 5, and the user shall be able to preview it in the application, as shown in Figure 2c, or view it outside the application, showing the resource in the corresponding application to that resource. The patient can rate both resources of Figures $2 a$ and $2 b$, using a rank mechanism based on stars.

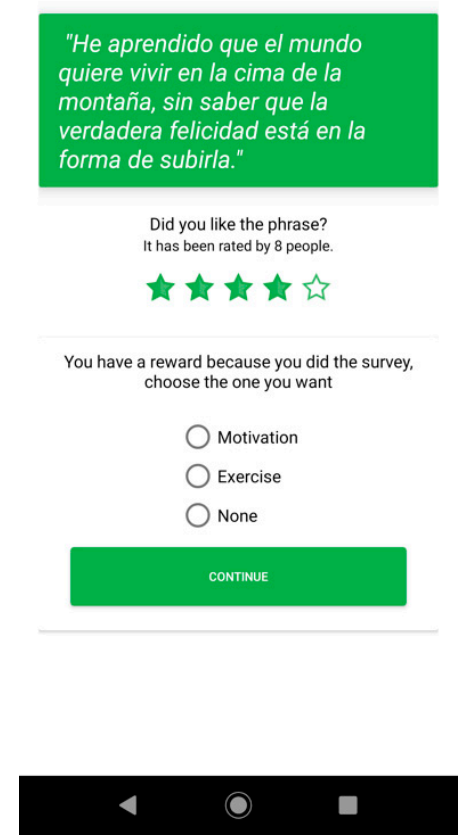

(a)

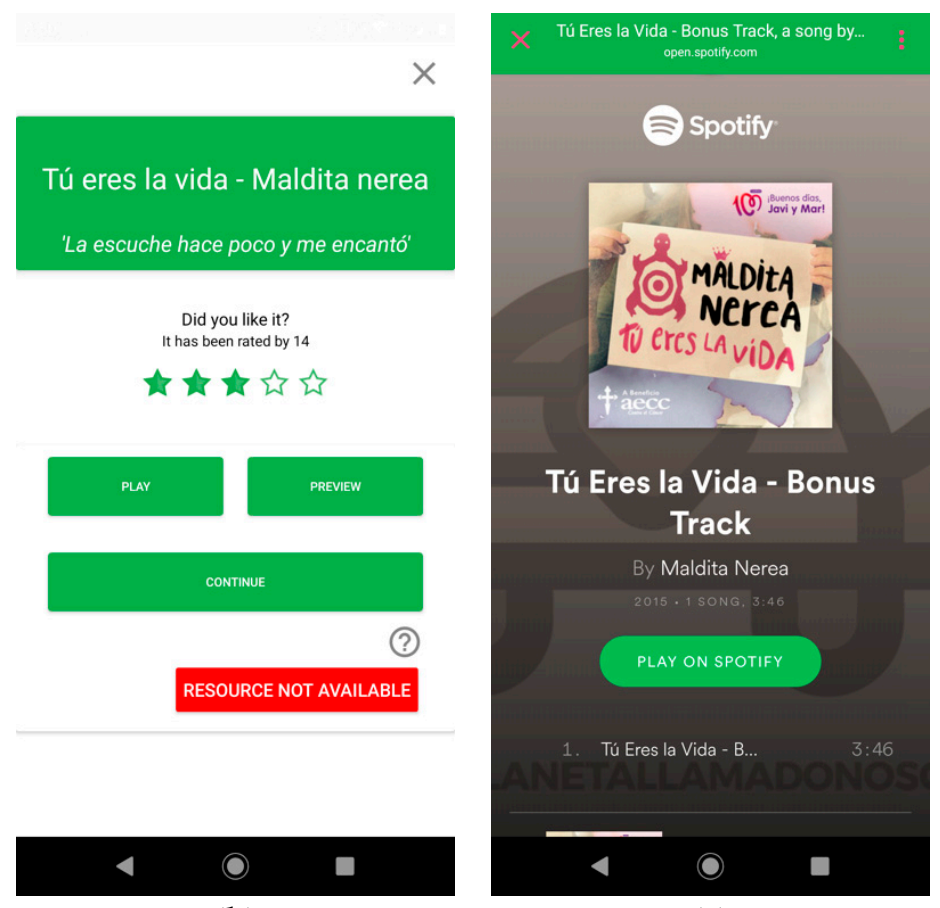

(b)

(c)

Figure 2. Screens of the user interface: (a) Reward after completing a survey, (b) Motivation reward and (c) Reward preview

If the patient selects an exercise, the screen of Figure 3a will be shown, in which the patient will visualize a message with the instruction to be performed. He will have the option of moving on to 
the next or previous instruction. When a patient reaches the end of a repetition, the system will show him the missing ones and a button to repeat, as seen on Figure $3 b$. The user is allowed to finish the exercise before doing all the repetitions, but in that case, he is asked why he did not finish it. In this way, we want the psychologists to see if the problem comes from the patient (e.g., health status, tiredness or pain), or from the exercise (e.g. long, boring or too many repetitions), so that they have that information in order to improve their monitoring or just to see if it is necessary to refine the exercise, improving it for other patients.

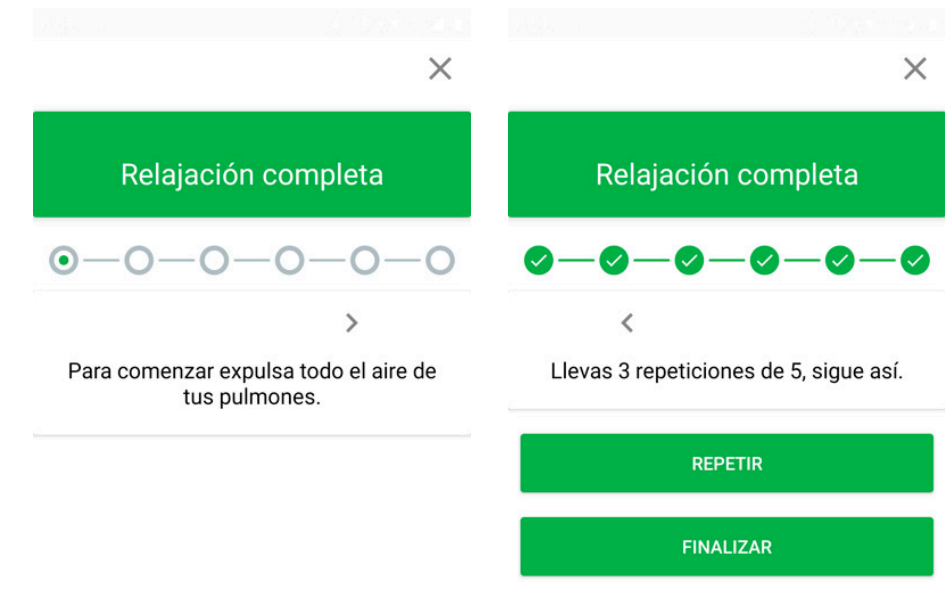

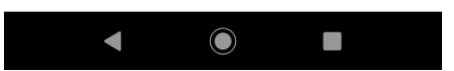

(a)

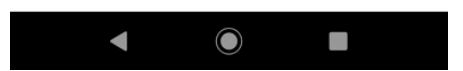

(b)

Figure 3. Screens of the user interface: (a) Exercise reward first screen and (b) Exercise reward when the patient had to do a repetition

To complete the part of the gamification, there is a screen in which patients can see both their latest motivation item and their last exercise (Figure 4a), so the patient can see it again if he wants, or if he did not have time enough to evaluate it. It is also shown whether the resource has already been valued or not, since they can only be assessed once. From this screen you can also access to see your points as you can see in Figure $4 \mathrm{~b}$. The user will see his classification, the total points earned, and the points needed to reach the next level. The levels are (a) bronze, (b) silver and (c) gold.

The share button on the screen, as seen in Figure 4a, will take the user to the screen for sharing with Close2U (Figure 4c), where you can choose a category and enter a name for the motivation, a description and a URL, except in the phrase category, where the only possibility is to enter a phrase. We want the patient to be able to share anything that comes him to mind, either while watching a video on YouTube, listening to a song in Spotify, a podcast, reading, etc. In this way they can copy the link and paste it into the share with Close2U screen after choosing a category, but we wanted to make it easier and implement the share option that any Android application has. Thus, our application can be seen as an option to share with, as shown in Figure 5a, and so, the URL is automatically copied into our app. In the screen of Figure $5 \mathrm{~b}$, just a name and a description of the resource have to be provided in order to share it. 


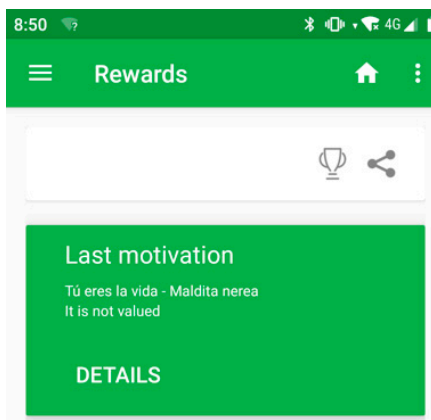

Last exercise

Relajacion completa

DETAILS
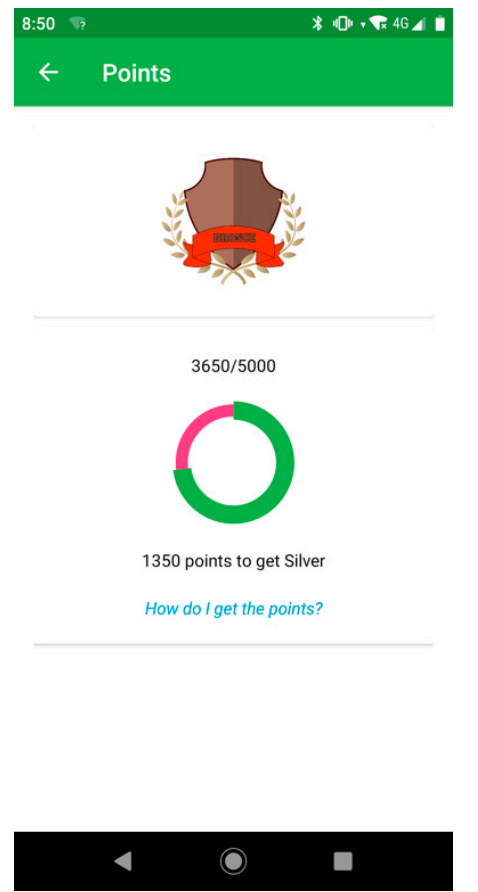

(b)

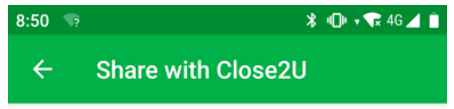

Help us by sharing what you like so that you can use it as rewards, which you can get by doing surveys. Choose the type of reward you want to
share.

IMAGE PHRASE

OTHER

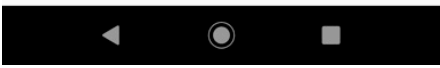

(a)

Figure 4. Screens of the user interface: (a) Rewards screen, (b) Points screen and (c) Share screen.

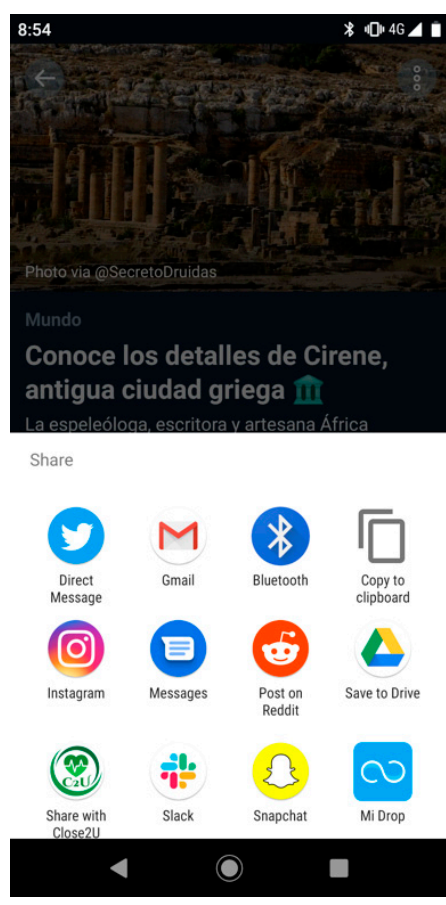

(a)

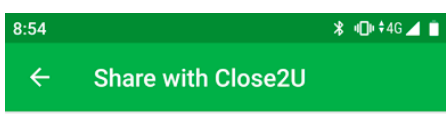

$<$ OTHER

Name:

News

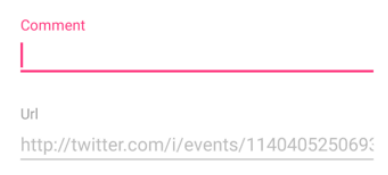

(2)
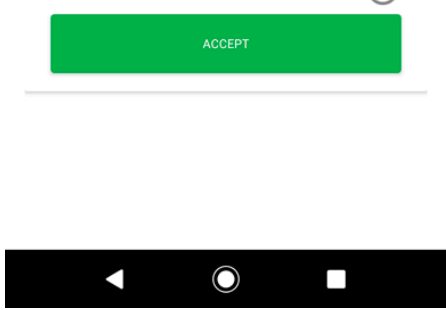

(b)

Figure 5. Screens of the user interface: (a) Share with Close2U in other application and (b) Share a resource with the URL taken from share option from other application.

From the web application, psychologists can see the resources that patients upload (Figures 6 and 7), can see the rating and the number of votes that a given resource has, the status of this resource (i.e. activated or deactivated), and they can eliminate, activate or deactivate it. Regarding the motivational resources that are not phrases, they will be able to visualize what the patients have shared and see if it is adequate to be shown to other patients. 


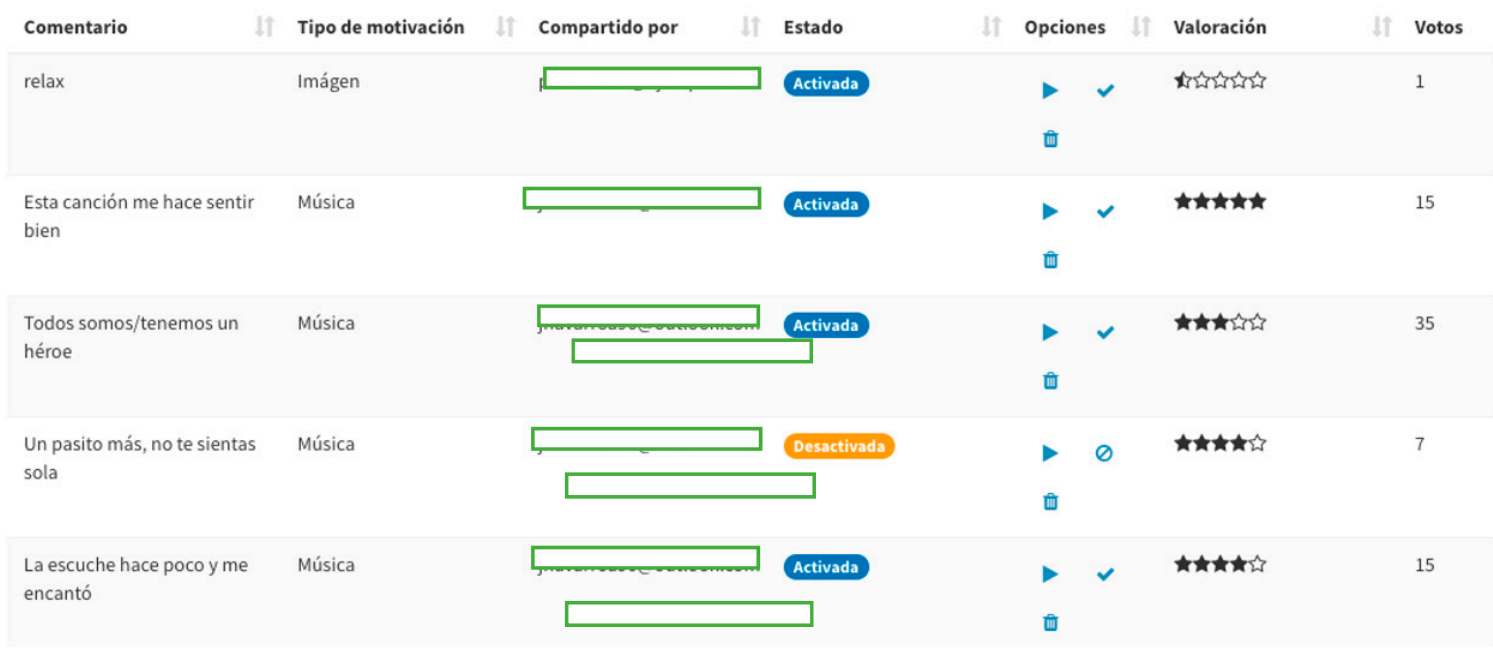

Figure 6. Screens of the web interface where the psychologists can see and manage the motivations.

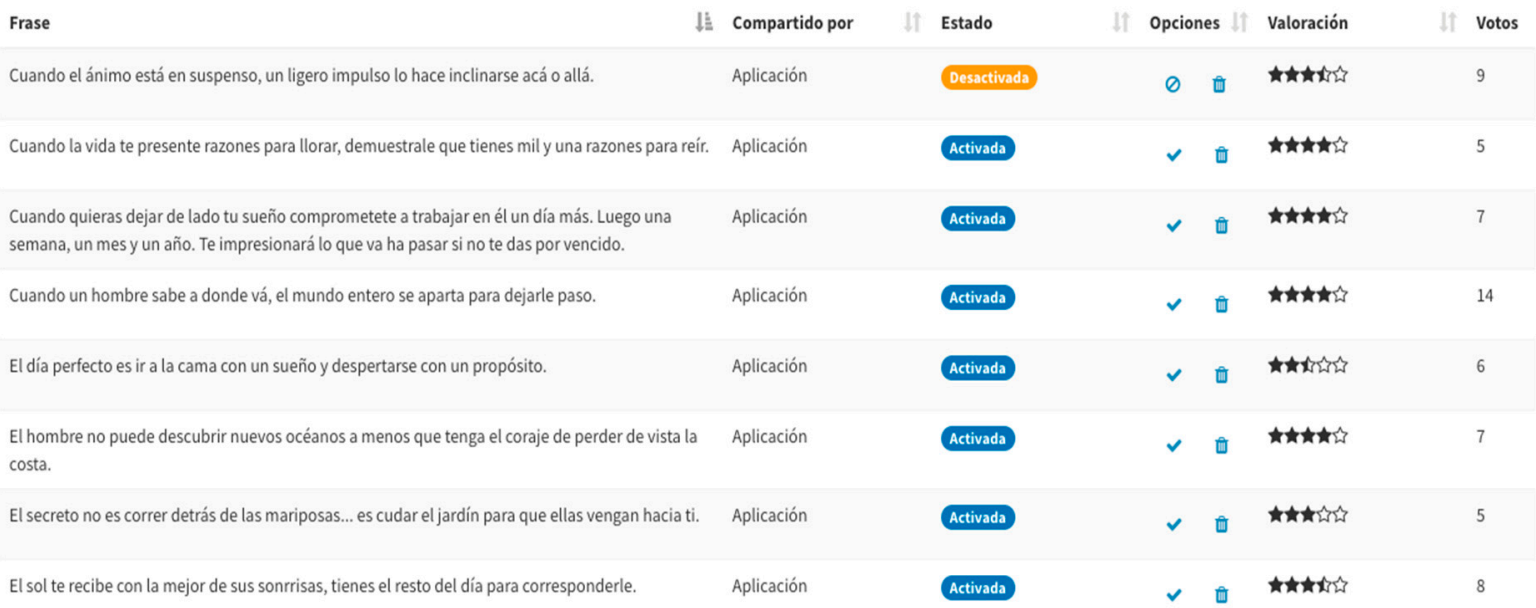

Figure 7. Screens of the web interface where the psychologists can see and manage the phrases.

The psychologist can create or modify exercises as it is shown in Figure 8, defining a name and a number of repetitions. Steps can be added one by one with the add step button or by entering a number of steps and pressing the Add button in the text field Add steps. In the same way, we allow to remove the steps all at once with the Clean steps button or to remove them one by one from the last step added with the Remove step button.

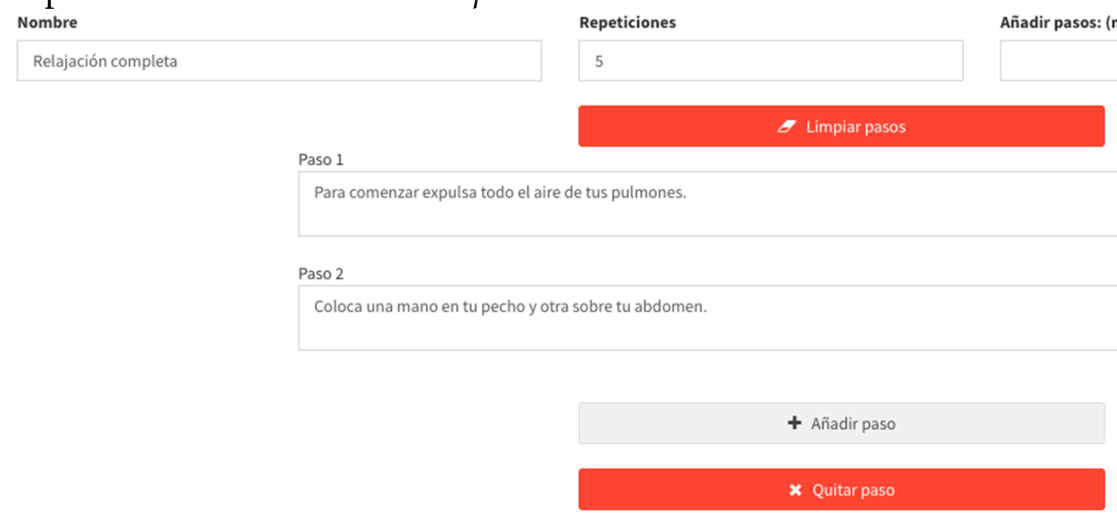

Figure 8. Screens of the web interface where the psychologists can manage the exercises. 


\section{Validation}

This section describes the study conducted to evaluate the gamification part of the Close2U application, introducing the sample of participants and the measurement methods.

\subsection{Participants}

There were 15 users that voluntarily participated in this study, without any economical compensation. They were 40.53 years old in average with a standard deviation (SD) of 16.85 . They were 7 males and 8 females. All of them were cancer patients or cancer survivors.

\subsection{Measures}

\subsubsection{App Usability and Ease of Use}

We used the System Usability Scale (SUS) [15] to measure the usability, which is measured with a questionnaire with 10 items. We also used the Usefulness, Satisfaction and Ease of Use (USE) [16] to measure the ease of use, which measures four dimensions: (a) Usefulness (8 questions), (b) Ease of use (11 questions), (c) Ease of learning (4 questions) and (d) Satisfaction (7 questions).

We urge participants to fundamentally value the motivation part in the use of the application, ranking the system, motivational resources, the exercises and the resource-sharing functionality.

The average results of SUS were in the 1-5 range and the average results of USE were in the 1-7 range. We converted these results to the same range of 0-100 for the SUS and USE questionnaires, in order to be able to easily compare both results.

\subsubsection{App Gamification}

We wanted to know if our way of applying the gamification in our application was appropriate and useful, so we created a survey with the following questions:

1. Prioritize the aspects that most motivate you in the use of the application.

2. Other aspects or ideas that motivated you to use the application more often.

3. Did the phrases, motivational resources and exercises at the end of the surveys motivate you to continue doing the surveys?

4. Did it motivate you to have the option to upload resources and share them with other users of the application?

5. Did you find interesting that your resources were evaluated and obtained points of contribution?

6. Do you think the way of sharing resources was intuitive?

Item 1 was replied by ordering the following aspects: ranking of points, share resources, do exercises, obtaining resources, obtaining medals/level up and others. Item 2 was replied with an optional text, in order to get the feedback of their ideas for news motivations or ways that we can improve the application. Finally, Items 3, 4, 5 and 6 were replied with a five-point Likert scale.

\section{Results and Discussion}

Regarding usability, the average result of SUS test was 72.67 in the range of $0-100$, and the standard deviation (SD) was 10.83. The average result of USE test was 80.64 and the SD was 15.08. Figure 9 shows the results of both questionnaires in order to compare the results of usability and the three dimensions of the USE questionnaire (Ease of use dimension is separated into two factors, Ease of Learning and Ease of Use). 


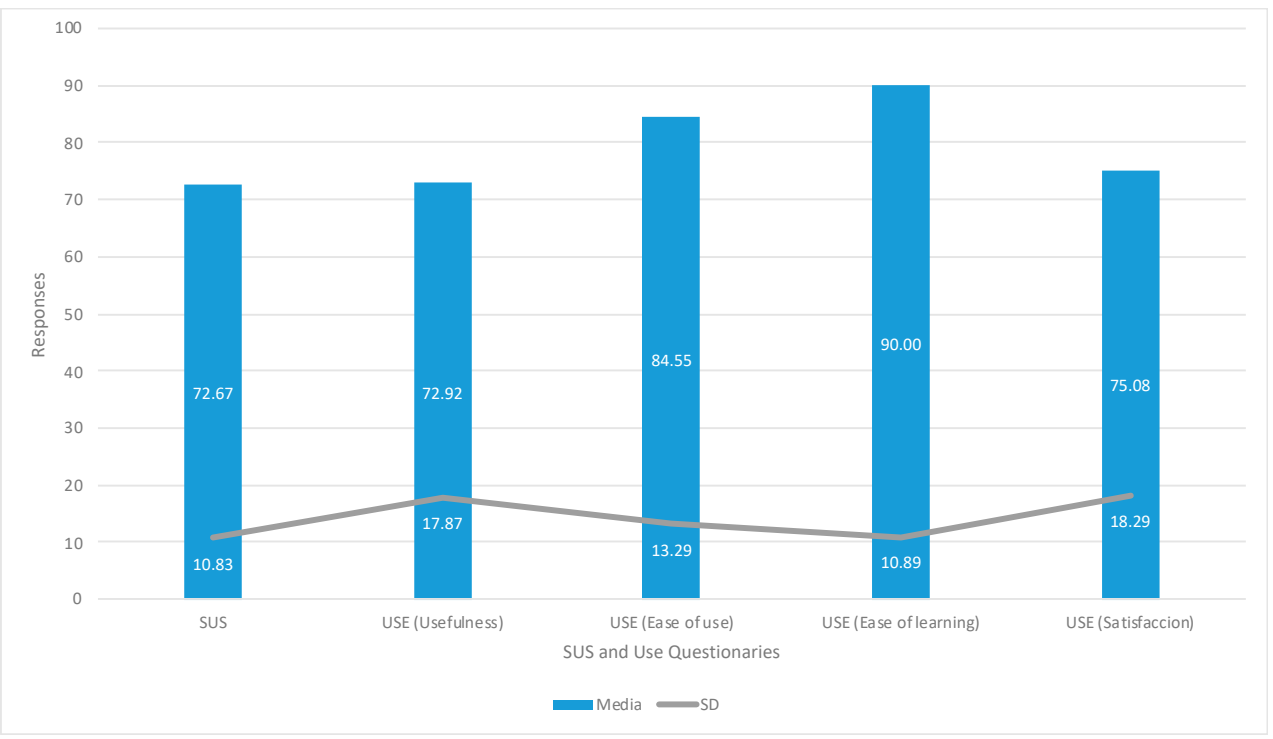

Figure 9. Relation of SUS and USE questionnaires.

We asked the participants some questions about how they felt using the application in terms of the gamification part. Table 2 shows a ranking with the aspects that most motivated them in order to use the application.

Table 2. Results for the first question for the gamification questionnaire.

\begin{tabular}{ccccccc}
\hline & $\begin{array}{c}\text { Ranking of } \\
\text { Points }\end{array}$ & $\begin{array}{c}\text { Share } \\
\text { Resources }\end{array}$ & $\begin{array}{c}\text { Do } \\
\text { Exercises }\end{array}$ & $\begin{array}{c}\text { Obtain } \\
\text { Resources }\end{array}$ & $\begin{array}{c}\text { Obtain } \\
\text { Medals/Level up }\end{array}$ & Others \\
\hline Average & 3 & 1 & 1 & 2 & 4 & 6 \\
SD & 1.51 & 1.64 & 1.55 & 1.12 & 1.41 & 1.30 \\
\hline
\end{tabular}

Table 3 shows the results of the questions replied with a 5-point Likert scale in our questionnaire about the gamification part.

Table 3. Results of the questions replied with 5-point Likert scale for the gamification questionnaire.

\begin{tabular}{ccccc}
\hline & Question 3 & Question 5 & Question 6 & Question 7 \\
\hline Average & 4.20 & 3.93 & 3.87 & 3.93 \\
SD & 1.29 & 1.30 & 1.20 & 1.49 \\
\hline
\end{tabular}

In the results, we observed that users were mostly interested in sharing resources and doing exercises, followed by obtaining resources. On the other hand, in the 5-point Likert answers of the gamification questionnaire, one can observe that users found interesting that resources could be valued and thus obtain points or simply the fact of sharing them so that other users can be motivated. They also valued in a very positive way that the rewards were given after completing each survey, and that fact motivated them to continue doing it with assiduity. The way to share the resources was also valued very positively. In the answers to the question about what type of resources motivated them the most when conducting the survey and monitoring, the answers were very varied such as: the songs recommended as motivation, videos, photos, exercises, music and videos. Thus, the results advocate that the rewarding mechanism works, thanks to what they share. Thus, the more they share in the future the better, since there will be more rewards that will motivate them.

The results of descriptive statistical analysis indicate that participants considered the Close2U application (a) to be both easy to use and easy to learn and (b) to have useful information useful. The highest rate was the ease of use dimension. We consider this dimension essential so that all the patients know how to use the application and carry out the task of the app without needing technical 
support. In both SUS and USE questionnaires, values of standard deviation indicated that the participants' responses were highly consistent.

\section{Conclusions and Future Work}

A mobile application has been developed that performs a complete follow-up of the patient. This article has focused on creating a mechanism to engage patients and make them use the application periodically for their own well-being, by means of gamification methods.

We can see that we have implemented a scalable and self-recursive system, which will allow patients to create their own resources with a high degree of motivation since they will be shared by other patients in their same situation. In this way, patients actively contribute to their follow-up and the more they do it, the more their engagement increases, having more motivational resources and rewards.

We have created a gamification system that helps patients to be motivated and fulfill their follow-up records. According to the studies carried out, when they have to carry out surveys on paper, they end up getting tired and do not get to perform them. The gamification system has been tested, being satisfactory for the users and allows them to contribute in their own community. One of the relevant factors of our application is the incorporation of social interaction among patients. The results show that the proposed approach works, and increases the cancer patients' motivation.

In the future, one of the main objectives of the project is to perform a non-invasive recording and measurement of the evolution of patients, using devices or sensors to improve both their treatment and their quality of life. In this way, we will have more options to give points to patients either by putting achievements by distance travelled, steps by day/week, or simply by using the devices regularly along with the application. Furthermore, we want to work with oncologists and develop a way in which the application can address the distinctive features of each type of cancer. With some of the feedback that we have achieved we know that we need to improve the ranking system and the way that the patients perceive the received points.

Funding This work has been funded by the research projects "New technologies in cancer monitoring and treatment" and "Advances in research, diagnosis and monitoring of bone cancer patients as a primary or secondary tumor", by the University Foundation "Antonio Gargallo". This work has also been partly funded by the Spanish Science, Innovation and University Ministry (MCIU), the National Research Agency (AEI) and the EU (FEDER) through the contract RTI2018-096986-B-C31 and by the Aragonese Government and the EU through the FEDER 2014-2020 “Construyendo Europa desde Aragón” action (Group T25_17D).

Acknowledgments: We would like to thank the psychologists of the AECC of Teruel for their willingness to help us in improving the application.

Conflicts of Interest: The authors declare no conflict of interest.

\section{References}

1. Deterding, S.; Sicart, M.; Nacke, L.; O'Hara, K.; Dixon, D. Gamification. using game-design elements in non-gaming contexts. In Proceedings of the CHI'11 Extended Abstracts on Human Factors in Computing Systems, Vancouver, BC, Canada, 7-12 May 2011; pp. 2425-2428.

2. Sardi, L.; Idri, A.; Fernández-Alemán, J.L. A systematic review of gamification in e-Health. J. Biomed. Inform. 2017, 71, 31-48.

3. Kawachi, I. It's all in the game-The uses of gamification to motivate behavior change. JAMA Internal Med. 2017, 177, 1593-1594.

4. Johnson, D.; Deterding, S.; Kuhn, K.A.; Staneva, A.; Stoyanov, S.; Hides, L. Gamification for health and wellbeing: A systematic review of the literature. Internet Interv. 2016, 6, 89-106.

5. Loibl, S.; Lederer, B. The importance of supportive care in breast cancer patients. Breast Care 2014, 9, 230.

6. Primack, B.A.; Carroll, M.V.; McNamara, M.; Klem, M.L.; King, B.; Rich, M.; Chan, C.W.; Nayak, S. Role of video games in improving health-related outcomes: a systematic review. Am. J. Prev. Med. 2012, 42, 630-638.

7. Cugelman, B. Gamification: what it is and why it matters to digital health behavior change developers. JMIR Serious Games 2013, 1, e3.

8. Marczewski, A. Even Ninja Monkeys Like to Play; Blurb Inc.: London, UK, 2015. 
9. Economou, D.; Dwek, M.; Roberston, C.; Elliott, B.; Kounenis, T.; Azimi, T.; Ramezanian, M.; Bell, N. PhytoCloud: A gamified Mobile Web Application to modulate diet and physical activity of women with breast cancer. In Proceedings of the 2017 IEEE 30th International Symposium on Computer-Based Medical Systems (CBMS), Thessaloniki, Greece, 22-24 June 2017; pp. 684-689.

10. SickKids Research. Available online: https://lab.research.sickkids.ca/iouch/pain-squad-app/ (accessed on 28 June 2019).

11. Mango Health. Available online: www.mangohealth.com (accessed on 28 June 2019).

12. Kinect para Windows. Available online: https://developer.microsoft.com/es-es/windows/kinect (accessed on 28 June 2019).

13. Reflexion Health. Available online: https://reflexionhealth.com/ (accessed on 28 June 2019).

14. Kim, B. Designing Gamification in the Right Way. Libr. Technol. Rep. 2015, 51, 29-35.

15. Brooke, J. SUS-A quick and dirty usability scale. Usability Eval. Ind. 1996, 189, 4-7.

16. Lund, A.M. Measuring usability with the use questionnaire12. Usability Interface 2001, 8, 3-6.

(C) 2019 by the authors. Licensee MDPI, Basel, Switzerland. This article is an open access article distributed under the terms and conditions of the Creative Commons Attribution (CC BY) license (http://creativecommons.org/licenses/by/4.0/). 